\title{
MEDICIONES DE CAUDAL LÍQUIDO Y SÓLIDO EN CUENCAS URBANAS DE ZONA SERRANA DE MISIONES
}

\author{
Tomás Rodriguez ${ }^{(*)}$ y Juan Sanchez \\ Laboratorio de Ingeniería Civil, Facultad de Ingeniería, Universidad Nacional de Misiones. Oberá, Misiones, \\ Argentina. \\ ${ }^{(*)}$ e-mail: tomas8448@hotmail.com
}

\begin{abstract}
RESUMEN
La falta de datos confiables y precisos es uno de los principales obstáculos para la correcta aplicación de tecnologías apropiadas en los modernos sistemas de drenaje urbano. Particularmente, la provincia de Misiones presenta un marcado déficit de información hidroambiental de sus cursos de agua internos. Déficit que se hace más evidente, en la información discretizada en intervalos de tiempo subdiarios como los que son necesarios en la modelación de cuencas urbanas de cabecera. El presente trabajo presenta la aplicación de una serie de metodologías para registrar aforos de variables hidrosedimentológicas, en una cuenca urbana de la ciudad de Oberá, Misiones. Las mediciones realizadas se presentan como inéditas en la región misionera de estudio, caracterizada por una topografía serrana de altas pendientes, con suelos finos del tipo limoarcillosos y clima subtropical húmedo donde se desarrollan precipitaciones intensas. Las mediciones alcanzadas representan rangos relativamente amplios de registros, los que cubren un significativo espectro de valores. De los 10 eventos registrados, la precipitación total varió entre 31 y $160 \mathrm{~mm}$, los caudales líquidos máximos entre 7 y 69 m³ y las máximas concentraciones de sólidos entre 369 y 1602 PPM. Los aforos registrados han sido utilizados en una etapa posterior en la calibración de modelos hidrológico-hidráulicos, acoplados a modelos de producción de sedimentos por erosión hídrica ante eventos lluviosos en cuencas urbanas de la región serrana de Misiones.
\end{abstract}

Palabras clave: Mediciones; caudal líquido; caudal sólido; cuencas urbanas; zona serrana; Misiones.

\begin{abstract}
The lack of reliable and accurate data is one of the main obstacles to the correct application of appropriate technologies in modern urban drainage systems. In particular, the province of Misiones presents a marked deficit of hydro-environmental information of its internal water courses. Deficit that becomes more evident, in discretized information in subdaily time intervals such as those that are necessary in the modeling of urban headwaters basin. This work presents the application of a series of methodologies to register indicators of hydrosedimentological variables, in an urban basin of the city of Oberá, Misiones. The measurements are presented as unprecedented in the mountain region of Misiones, characterized by mountain topography of steep slopes, with fine soils of silt-clayey type and humid subtropical climate where intense precipitations develop. The measurements reached represent relatively wide ranges of records, which cover a significant range of values. With a total of 10 registered events, the total precipitation varied between 3 and $160 \mathrm{~mm}$, the maximum flow rates between 7 and $69 \mathrm{~m}^{3} / \mathrm{s}$ and the maximum solids concentrations between 369 and 1602 PPM. The registered have been used at a later stage in the calibration of hydrological-hydraulic models, coupled to sediment production models by water erosion in rainfall events in urban basins of the mountain region of Misiones.
\end{abstract}

Keywords: Measurements; liquid flow; solid flow; urban basins; mountain area; Misiones 


\section{INTRODUCCION}

En ambientes urbanos, durante largo tiempo, la solución del control de los excedentes hídricos superficiales se ha encarado a través de la ejecución de obras de drenaje, para remover el agua pluvial del área urbana de la forma más eficiente posible. La experiencia indica que las soluciones más convenientes se alcanzan a partir de una compresión más integrada de los procesos asociados al escurrimiento de los excedentes hídricos.

A partir de la década del ' 70 , en los países desarrollados se fue extendiendo el concepto del manejo del agua pluvial urbana, como la percepción de un conjunto planificado de medidas estructurales y no estructurales destinadas a administrar el agua pluvial de una manera integral, racional y sustentable. Bajo este concepto, el drenaje es concebido como un subconjunto del manejo e interrelacionado con otros propósitos, tales como la regulación de crecidas, el mejoramiento de la calidad de agua pluvial, el control de la erosión, la creación o conservación de espacios abiertos para recreación y otros usos.

Sin embargo, los problemas concretos de cada sitio requieren soluciones específicas. Las tecnologías modernas de los países desarrollados no pueden ser copiadas simplemente, ya que no funcionan correctamente al ser implementadas en zonas para las que no fueron diseñadas.

A su vez, para la aplicación de adecuadas técnicas y tecnologías disponibles para la gestión del agua de lluvia, es necesaria la descripción de los procesos hidrológicos y sedimentológicos involucrados en la generación y transporte del escurrimiento superficial, variables tales como niveles, velocidades, caudales y concentración de sólidos en cada elemento del sistema de desagüe pluvial.

Para el diagnóstico de la situación del sistema existente, la implementación de modelos avanzados (lluvia-escorrentía) y la evaluación del efecto sobre las superficies y cuerpos de agua receptores locales, tienen que ser recogidos datos específicos y los modelos deben ser adaptados.

Se ha señalado muchas veces (Dunne, 1986; Maksimović, 1992) que la falta de datos confiables y precisos es uno de los principales obstáculos para la correcta aplicación de tecnologías apropiadas en los modernos sistemas de drenaje urbano.
Particularmente, la descripción de la generación, traslado y disposición de sólidos en ambientes urbanos presenta un alto grado de complejidad dada la gran cantidad de fuentes y materiales susceptibles de ser incorporados a la corriente hídrica. Además de los suelos erosionados por flujo mantiforme en las áreas permeables y el flujo concentrado en los cursos de agua, se suman los sedimentos generados por las actividades humanas, depositados en superficies impermeables como calles, veredas, techos y solados de edificios.

En zonas como la de estudio, además de las afectaciones negativas sobre el medioambiente por procesos de erosión, los suelos finos son susceptibles a la absorción, traslado y acumulación de contaminantes, pudiendo provocar impactos negativos crónicos en los cuerpos de agua receptores del desagüe pluvial y el ambiente en general.

Si bien se han encontrado estudios científicos sobre el comportamiento de las cuencas hidrológicas en Misiones, las mediciones hidroambientales de las mismas se presentan como deficientes para la concreción de estudios científicos de relevancia. Dicha escasez de datos se ve incrementada en lo referente a las cuencas urbanas de cabecera en la región serrana de la provincia.

El presente trabajo presenta la aplicación de una serie de metodologías para registrar aforos de variables hidrosedimentológicas, en una cuenca urbana de la ciudad de Oberá, Misiones (Figura 1). En particular se recabaron registros de precipitaciones, caudales líquidos y sólidos. Las mediciones realizadas se presentan como inéditas en la región misionera de estudio, caracterizada por una topografía serrana de altas pendientes, con suelos finos del tipo limo-arcillosos y clima subtropical húmedo donde se desarrollan precipitaciones intensas.

\section{OBJETIVOS}

El objetivo del trabajo es presentar los resultados obtenidos en la aplicación de metodologías de medición de variables hidrosedimentológicas en la cuenca rural-urbana del arroyo Mbotaby (Oberá, Misiones). Los aforos registrados han sido utilizados en una etapa posterior en la calibración de modelos hidrológico-hidráulicos, acoplados a modelos de producción de sedimentos por erosión hídrica ante eventos lluviosos en cuencas urbanas 
de la región serrana de Misiones. En particular, los aforos se utilizaron para la simulación de la transformación lluvia-caudal y de los procesos de erosión hídrica superficial en modelos implementados en el entorno del SWMM 5.0ve (GMMF, 2005), a través de una constitución con un alto nivel de detalle (Rodriguez, 2018).

\section{CUENCA PILOTO}

La cuenca en estudio, donde se implementaron las mediciones, es la cuenca del Arroyo Mbotaby (arroyo de tábanos, en dialecto guaraní). El brazo principal de dicho arroyo nace al Este de la ciudad de Oberá, en la divisoria de aguas de las cuencas de los ríos Paraná y Uruguay (Figura 2). La cuenca Mbotaby recoge $40 \%$ de los excedentes hídricos superficiales del casco urbano de la ciudad de Oberá, y si bien posee una impermeabilidad total estimada de $23.9 \%$ (Rodriguez, 2018), la misma se presenta como unas de las cuencas más densamente pobladas en la región Serrana de Misiones.

El ambiente de modelación, producto de las tareas de relevamiento y análisis de datos disponibles hasta el año 2013, resultó de una extensión superficial de 976.7 ha, correspondiendo un $10.9 \%$ a áreas impermeables directamente conectadas, $13.0 \%$ a áreas impermeables no conectadas y $76.1 \%$ a áreas permeables.

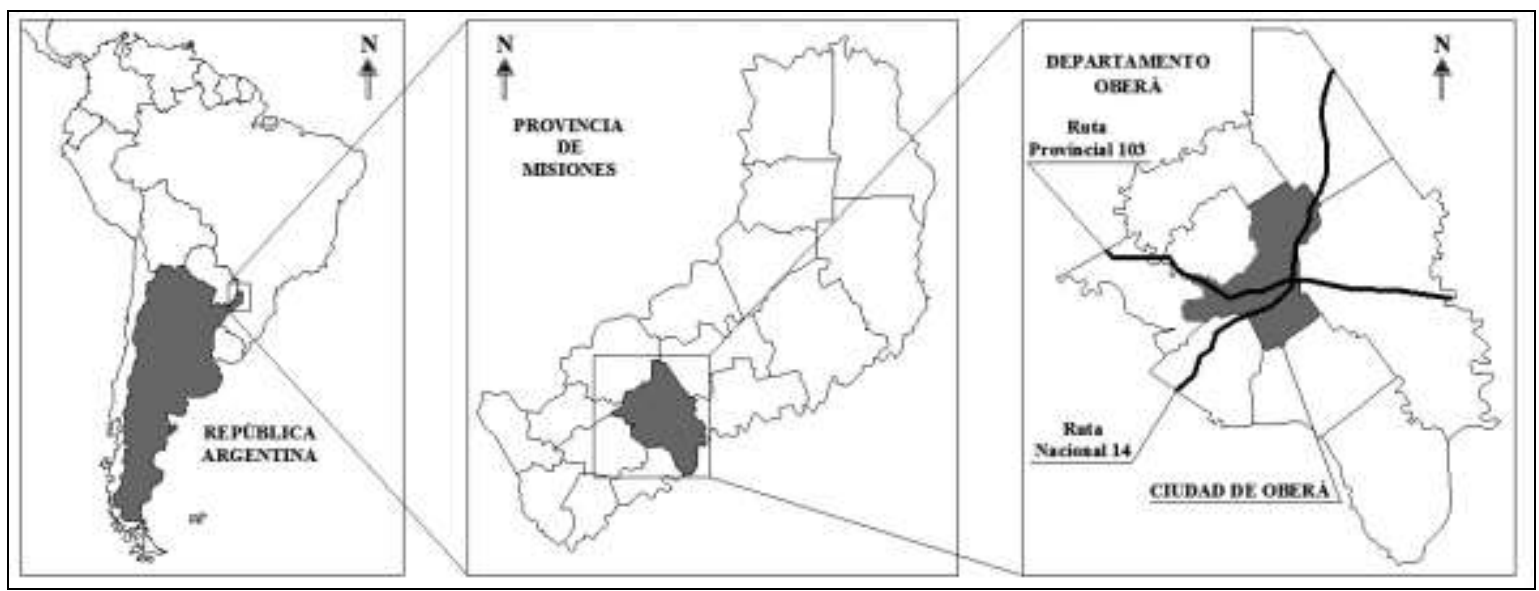

Figura 1. Ubicación geográfica de la ciudad de Oberá, Misiones, Argentina.

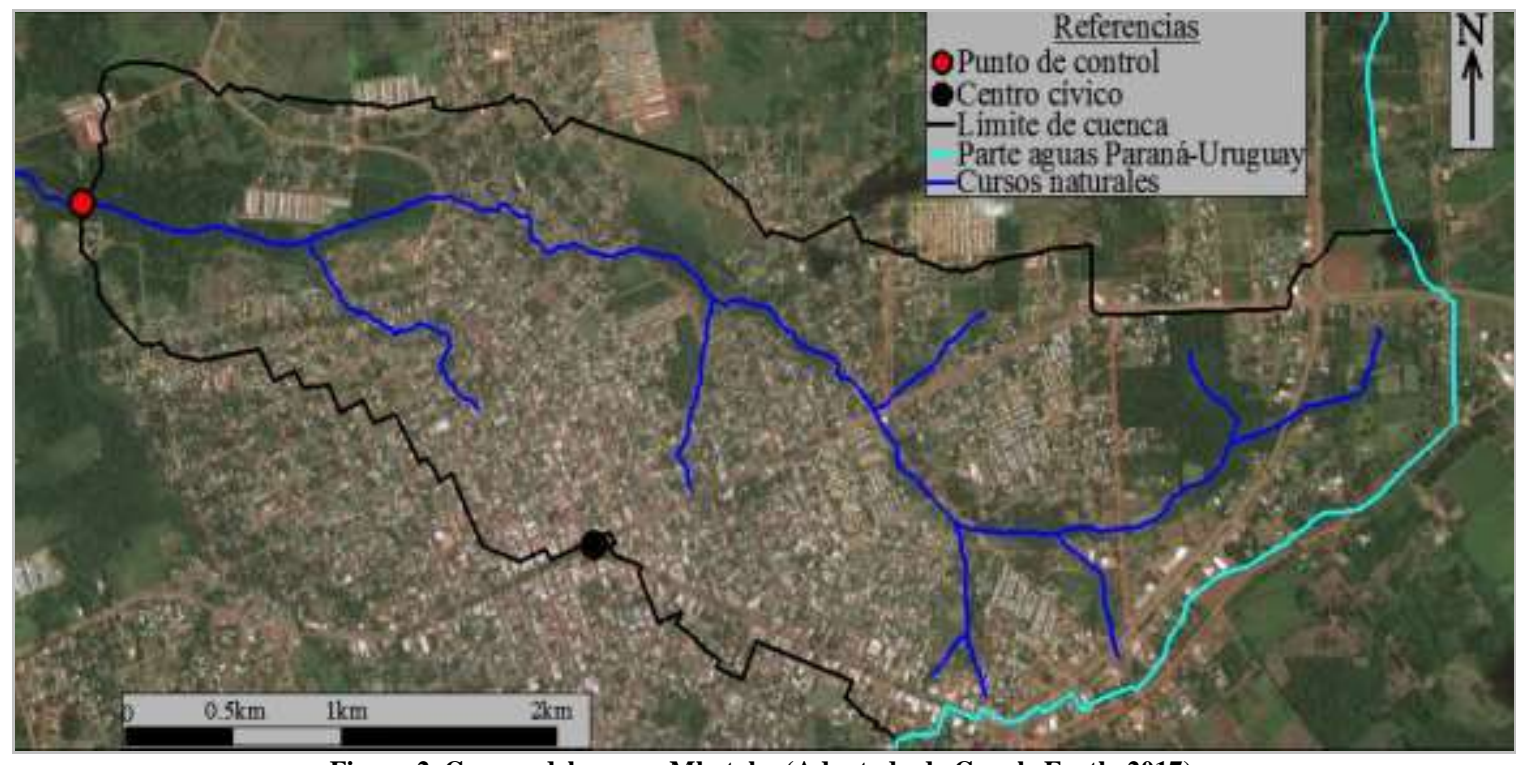

Figura 2. Cuenca del arroyo Mbotaby (Adaptado de Google Earth, 2017). 
La cuenca presenta en un $94.5 \%$ suelos del Complejo 9 (Tierra Colorada) caracterizada por un limo-arcilloso de coloración rojiza; mientras que el $5.5 \%$ restante de la superficie está cubierta por suelos del Complejo 6 (Suelos Pedregosos) compuestos por el basalto en descomposición (CARTA, 1964).

La región ha sido caracterizada como subtopical sin estación seca (Olinuk, 1998), con una distribución de precipitaciones a lo largo de todo el año y un módulo pluviométrico de $1925 \mathrm{~mm}$ (INTA Cerro Azul, 2013). Particularmente, según un informe del Servicio Meteorológico Nacional, la ciudad de Oberá fue la ciudad con mayores precipitaciones durante el 2017, con $2709 \mathrm{~mm}$ precipitados (SMN, 2018). Posee además, la distinción de estar sujeta a tormentas de gran intensidad, con importantes volúmenes precipitados en corto tiempo (Olinuk, 1998).

Respecto a las demás caracterizaciones hidrogeológicas referidas por Rodriguez (2018), la totalidad de la cuenca presenta una región natural de "Meseta Central Preservada" y un potencial de erosión "Moderadamente Alto".

\section{METODOLOGÍAS DE MEDICIÓN}

\section{Registro de hietogramas y limnigramas}

Los datos de precipitación fueron obtenidos por la estación meteorológica instalada en la Facultad de
Ingeniería de la Universidad Nacional de Misiones (FI-UNaM) $\left(27^{\circ} 29^{\prime} 06^{\prime \prime} \mathrm{S} ; 5^{\circ} 06^{\prime} 38^{\prime \prime} \mathrm{O}\right)$, situada en el campus universitario dentro del casco céntrico de la ciudad, cercana al baricentro de la cuenca en estudio.

Se trata de una estación digital con sensoriamiento remoto y almacenamiento automático de la información, lo que permite una discretización de las variables en el tiempo, tanto de las tormentas como de las demás variables meteorológicas registradas.

El registro de las precipitaciones se realiza mediante un pluviómetro digital a cangilones con $0.2 \mathrm{~mm}$ de precisión y con la posibilidad de discretizar los pulsos de lluvia cada 1 minuto.

Para la determinación del caudal erogado registrado se utilizó el limnigrama observado y la curva $Q(h)$ definida para el punto de control de la cuenca (Figuras 3.a y 3.b).

Los limnigramas comparados fueron obtenidos por observación directa de las reglas limnimétricas instalada en el punto de control de la cuenca.

\section{Obtención indirecta de hidrogramas}

La determinación de los caudales salientes y por ende de los volúmenes netos escurridos por la sección de control o de cierre de la cuenca se realizó en forma indirecta a partir de los limnigramas observados y la definición previa de una relación caudal-tirante.

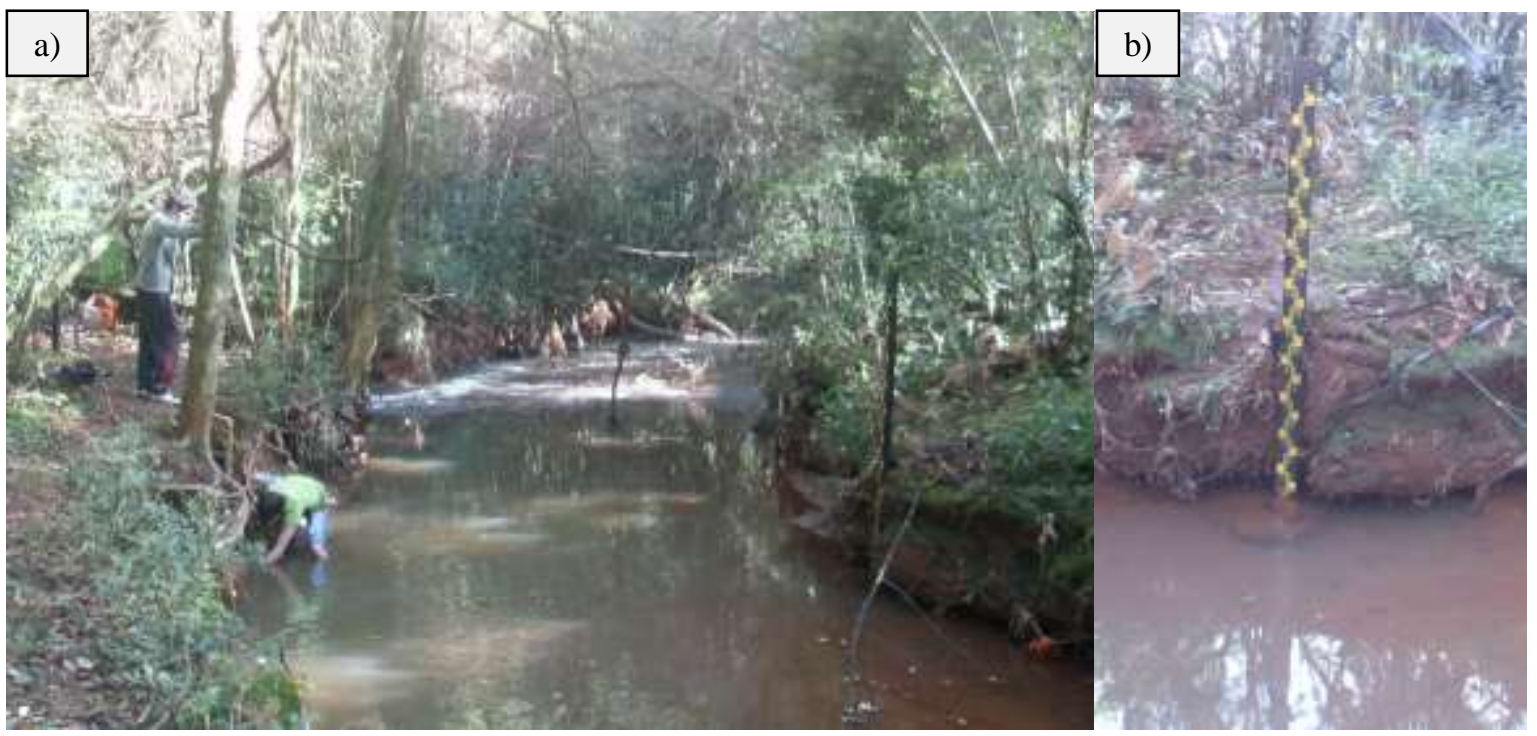

Figura 3. a) Sección de control utilizada para la calibración; b) Regla limnimétrica instalada para el registro de niveles. 
Producto de campañas de batimetría con relevamientos extendidos de la sección de control, en conjunto con mediciones de velocidades superficiales, se logró establecer la curva que relaciona los distintos tirantes en el punto de control con los caudales asociados, obteniéndose así la curva $Q(h)$ que permitió inferir los volúmenes totales erogados para cada tormenta registrada y compararlos con los determinados por el modelo.

Los pasos seguidos para determinar la $Q(h)$ fue:

1. Mediante la utilización de flotadores se registró el tiempo necesario para que los mismos recorran una distancia fija de $7.15 \mathrm{~m}$, para distintos niveles de agua en el arroyo.

2. La velocidad superficial fue estimada a partir de la relación $\Delta \mathrm{l} / \Delta \mathrm{t}$ considerando una distancia recorrida por el flotador de $7.15 \mathrm{~m}$. Los flotadores fueron arrojados de manera tal que su recorrido coincida con el thalweg (trayectoria a lo largo del cauce que une los puntos de mayor profundidad), por lo que la velocidad determinada corresponde a la máxima velocidad superficial $V_{\text {max }}^{s}$.

3. La velocidad media integrada en una vertical $V_{m}$ se estimó a partir de la relación $V_{m}$ $=0.857 \times V^{s}{ }_{\text {max }}$ (Basile et al., 2011).

4. El caudal inferido resulto de la multiplicación de $V_{m}$ por el área mojada en el cauce, establecida mediante le relevamiento planialtimétrico para cada nivel de agua registrado.

5. Finalmente la curva $Q(h)$ fue ajustada a los caudales registrados, mediante una ecuación potencial (Ecuación 1), la cual resulto con una $R^{2}$ de 0.99 .
$Q\left[m^{3} / \mathrm{s}\right\rfloor=6.4001 h[m]^{2.3499}$

Debido a las condiciones de riesgo que presenta el punto de control con el arroyo desbordado, no fue posible realizar mediciones de velocidades en todo el dominio de niveles registrados. El nivel máximo registrado en la totalidad de los eventos es de 2.75 $\mathrm{m}$, mientras que el nivel máximo en el que se pudo realizar mediciones de velocidad certeras es de 2.10 $\mathrm{m}$, recordando que el nivel de desborde del curso en el punto de control es de $1.80 \mathrm{~m}$. La Figura 4 presenta el perfil transversal de la sección de control y los niveles citados anteriormente.

En la Tabla 1 se observan los resultados de las campañas de mediciones superficiales de velocidad y batimetría, mientras que en la Figura 5 se presenta el ploteo de los caudales inferidos y la curva $Q(h)$ obtenida.

Cabe destacar que las campañas anteriormente descriptas permitieron además calibrar las rugosidades del cauce y el valle de inundación del arroyo donde se ubica la sección de control, los cuales fueron adoptados para todos los otros cursos naturales de agua.

\section{Determinación de sólidos en suspensión}

Además de efectuar el aforo de precipitaciones y niveles durante los eventos lluviosos, se realizó la toma de muestras de agua, lo cual posibilitó determinar la variación del TSD en el seno de la corriente hídrica de descarga de la cuenca a lo largo del desarrollo de las tormentas (Figura 6.a).

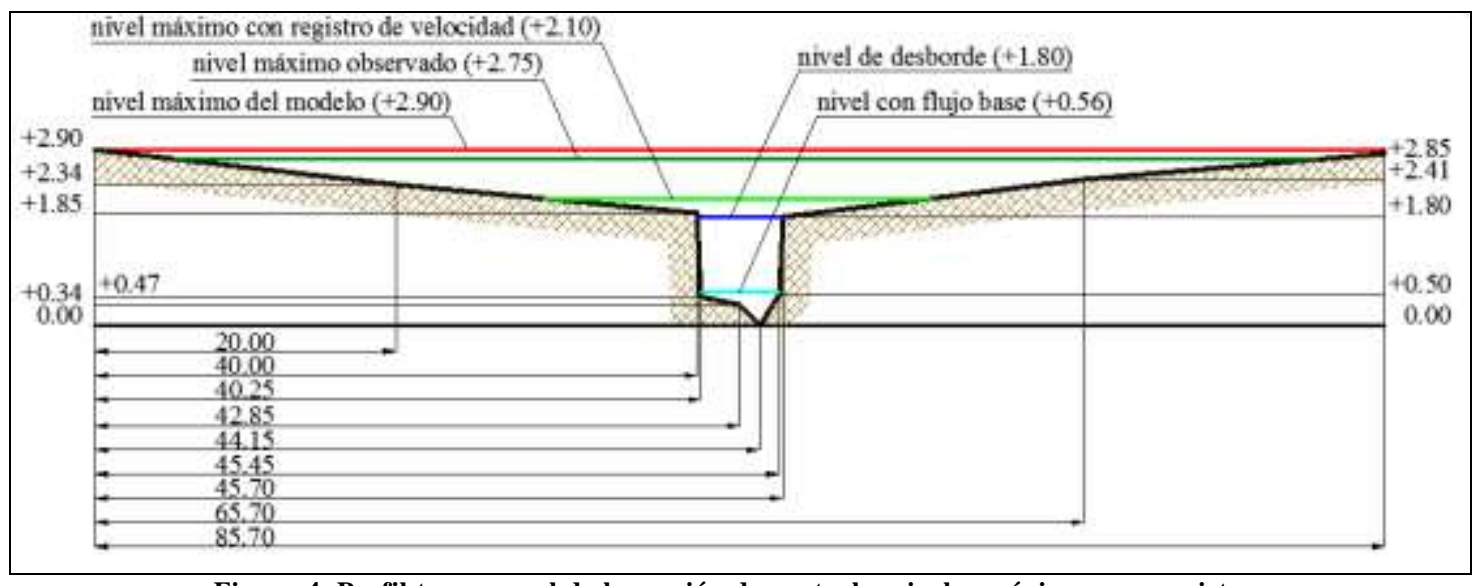

Figura 4. Perfil transversal de la sección de control y niveles máximos con registro. 


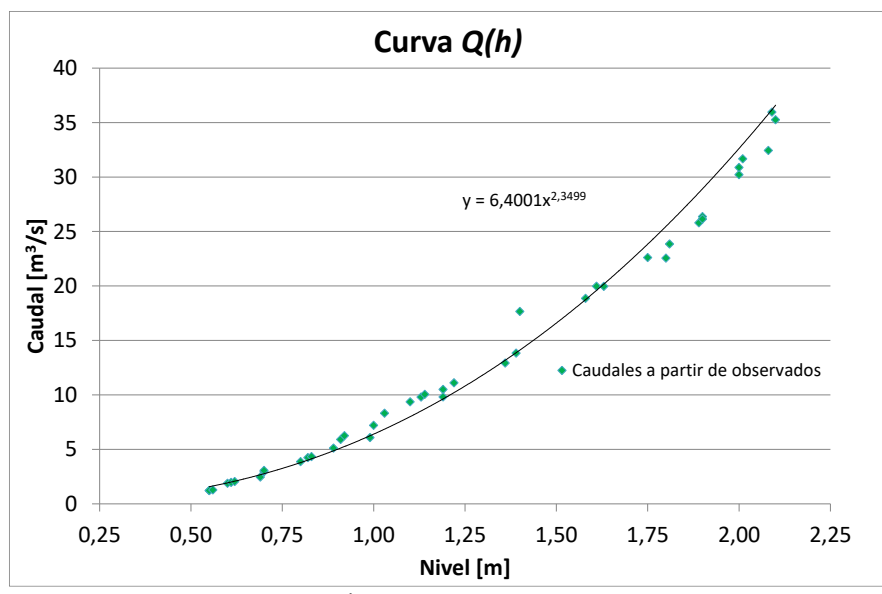

Figura 5. Curva de relación altura-caudal en la salida de la cuenca.

Tabla 1. Curva de relación altura-caudal en la salida de la cuenca.

\begin{tabular}{|c|c|c|c|c|c|c|c|}
\hline \multirow{2}{*}{ Observaciones } & nivel & sección & tiempo & velocidad superficial & velocidad media & caudal & curva $Q(h)$ \\
\hline & {$[\mathrm{m}]$} & {$\left[\mathbf{m}^{2}\right]$} & {$[\mathrm{s}]$} & {$[\mathbf{m} / \mathbf{s}]$} & {$[\mathrm{m} / \mathrm{s}]$} & {$\left[\mathrm{m}^{3} / \mathrm{s}\right]$} & {$\left[\mathrm{m}^{3} / \mathrm{s}\right]$} \\
\hline \multirow{31}{*}{ 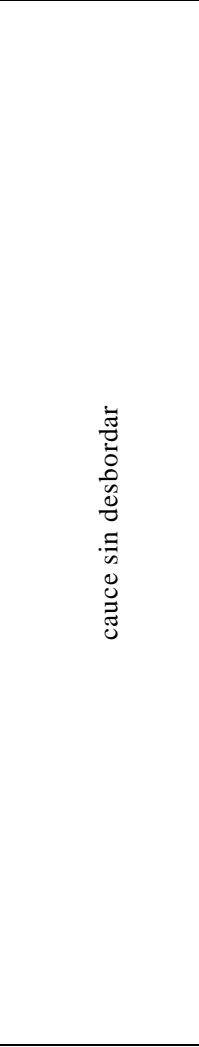 } & 0.55 & 1.26 & 6.39 & 1.12 & 0.96 & 1.21 & 1.57 \\
\hline & 0.55 & 1.26 & 6.35 & 1.13 & 0.97 & 1.22 & 1.57 \\
\hline & 0.56 & 1.31 & 6.32 & 1.13 & 0.97 & 1.27 & 1.64 \\
\hline & 0.60 & 1.52 & 4.95 & 1.44 & 1.24 & 1.89 & 1.93 \\
\hline & 0.61 & 1.58 & 4.91 & 1.46 & 1.25 & 1.97 & 2.00 \\
\hline & 0.62 & 1.63 & 4.87 & 1.47 & 1.26 & 2.05 & 2.08 \\
\hline & 0.69 & 1.94 & 4.86 & 1.47 & 1.26 & 2.45 & 2.68 \\
\hline & 0.70 & 2.05 & 4.28 & 1.67 & 1.43 & 2.94 & 2.77 \\
\hline & 0.70 & 2.05 & 4.10 & 1.75 & 1.50 & 3.07 & 2.77 \\
\hline & 0.80 & 2.58 & 4.09 & 1.75 & 1.50 & 3.87 & 3.79 \\
\hline & 0.82 & 2.63 & 3.81 & 1.88 & 1.61 & 4.24 & 4.01 \\
\hline & 0.83 & 2.69 & 3.80 & 1.88 & 1.61 & 4.33 & 4.13 \\
\hline & 0.89 & 3.01 & 3.60 & 1.99 & 1.70 & 5.12 & 4.87 \\
\hline & 0.91 & 3.17 & 3.29 & 2.18 & 1.87 & 5.91 & 5.13 \\
\hline & 0.92 & 3.22 & 3.16 & 2.26 & 1.94 & 6.25 & 5.26 \\
\hline & 0.99 & 3.54 & 3.57 & 2.00 & 1.71 & 6.08 & 6.25 \\
\hline & 1.00 & 3.65 & 3.11 & 2.30 & 1.97 & 7.20 & 6.40 \\
\hline & 1.03 & 3.81 & 2.81 & 2.55 & 2.18 & 8.32 & 6.86 \\
\hline & 1.10 & 4.19 & 2.75 & 2.60 & 2.23 & 9.36 & 8.01 \\
\hline & 1.13 & 4.30 & 2.69 & 2.66 & 2.28 & 9.79 & 8.53 \\
\hline & 1.14 & 4.35 & 2.66 & 2.69 & 2.31 & 10.05 & 8.71 \\
\hline & 1.19 & 4.63 & 2.70 & 2.65 & 2.27 & 10.50 & 9.63 \\
\hline & 1.19 & 4.63 & 2.89 & 2.47 & 2.12 & 9.81 & 9.63 \\
\hline & 1.22 & 4.85 & 2.67 & 2.67 & 2.29 & 11.11 & 10.21 \\
\hline & 1.36 & 5.62 & 2.66 & 2.68 & 2.30 & 12.92 & 13.18 \\
\hline & 1.39 & 5.73 & 2.54 & 2.82 & 2.41 & 13.83 & 13.88 \\
\hline & 1.40 & 5.84 & 2.16 & 3.53 & 3.03 & 17.66 & 14.11 \\
\hline & 1.58 & 6.84 & 2.22 & 3.22 & 2.76 & 18.86 & 18.75 \\
\hline & 1.61 & 7.01 & 2.15 & 3.32 & 2.85 & 19.97 & 19.60 \\
\hline & 1.63 & 7.07 & 2.17 & 3.30 & 2.83 & 19.96 & 20.17 \\
\hline & 1.75 & 7.80 & 2.12 & 3.38 & 2.90 & 22.60 & 23.84 \\
\hline \multirow{11}{*}{ 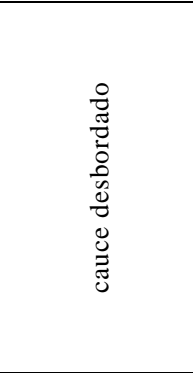 } & 1.80 & 8.08 & 2.20 & 3.26 & 2.79 & 22.56 & 25.47 \\
\hline & 1.81 & 8.16 & 2.10 & 3.41 & 2.92 & 23.85 & 25.81 \\
\hline & 1.89 & 8.71 & 2.07 & 3.45 & 2.96 & 25.79 & 28.57 \\
\hline & 1.90 & 8.87 & 2.06 & 3.47 & 2.97 & 26.37 & 28.92 \\
\hline & 1.90 & 8.87 & 2.08 & 3.44 & 2.95 & 26.14 & 28.92 \\
\hline & 2.00 & 10.34 & 2.10 & 3.41 & 2.92 & 30.21 & 32.63 \\
\hline & 2.00 & 10.34 & 2.05 & 3.48 & 2.99 & 30.87 & 32.63 \\
\hline & 2.01 & 10.56 & 2.04 & 3.50 & 3.00 & 31.67 & 33.01 \\
\hline & 2.08 & 12.10 & 2.29 & 3.13 & 2.68 & 32.44 & 35.78 \\
\hline & 2.09 & 12.32 & 2.10 & 3.40 & 2.92 & 35.95 & 36.18 \\
\hline & 2.10 & 12.54 & 2.18 & 3.28 & 2.81 & 35.25 & 36.59 \\
\hline
\end{tabular}


Los registros de niveles y tomas de muestra se realizaron inicialmente con intervalos de 1 hora, y una vez finalizo el escurrimiento por exceso de precipitación, cada 12 hs aproximadamente. Esto metodología se utilizó debido a dos factores: (i) dada la imposibilidad de acceder al punto de control de la cuenca durante la noche; (ii) debido a que una vez restituido el flujo base en el arroyo, el mismo evidenciaba un alto contenido de TSD.

Para realizar el análisis de las muestras de agua se recurrió a una metodología ampliamente utilizada por el Laboratorio de Hidráulica y el CURIHAM de la FCEIyA-UNR. La misma consta en determinar la cantidad de TSD mediante el secado en horno de las muestras de agua, según el procediendo detallado a continuación:

1. Por cada muestra de agua se debe pesar, en un balanza de precisión, tres recipientes resistentes al calor, limpios y numerados, de aproximadamente $300 \mathrm{ml}$ de capacidad.

2. De las muestras de agua originales, las cuales poseen un volumen aproximado de 1 litro, se separan tres submuestras de aproximadamente $150 \mathrm{ml}$, en los recipientes citados anteriormente.
3. Se pesan las submuestras obteniendo el valor corresponde a la suma de peso del recipiente, el agua propiamente dicha y el total de material solido (Figura 6.b).

4. Se produce el secado de la muestra en horno a $180{ }^{\circ} \mathrm{C}$, durante $24 \mathrm{~h}$.

5. Una vez enfriada la muestra se la pesa obteniendo el peso del recipiente más el total de sólidos (Figura 6.c).

6. El TSD de cada submuestra se determina en función a la Ecuación 2 según:

$T S D=\frac{P S-\operatorname{Pr}}{\text { Ptotal }-\operatorname{Pr}} 1.000 .000[\mathrm{ppm}]$

Donde: Pr: peso del recipiente seco; Ps: peso del recipiente más sólidos secos; Ptotal: peso del recipiente más la muestra total (agua y sólidos).

7. Finalmente es necesario realizar un análisis estadístico de manera de descartar aquellas submuentras que posean valores atípicos. El TSD de la muestra resultara del promedio de los valores de sus respectivas submuestras. En la Tabla 2 se presenta un ejemplo de cálculo del TSD para una muestra de agua.

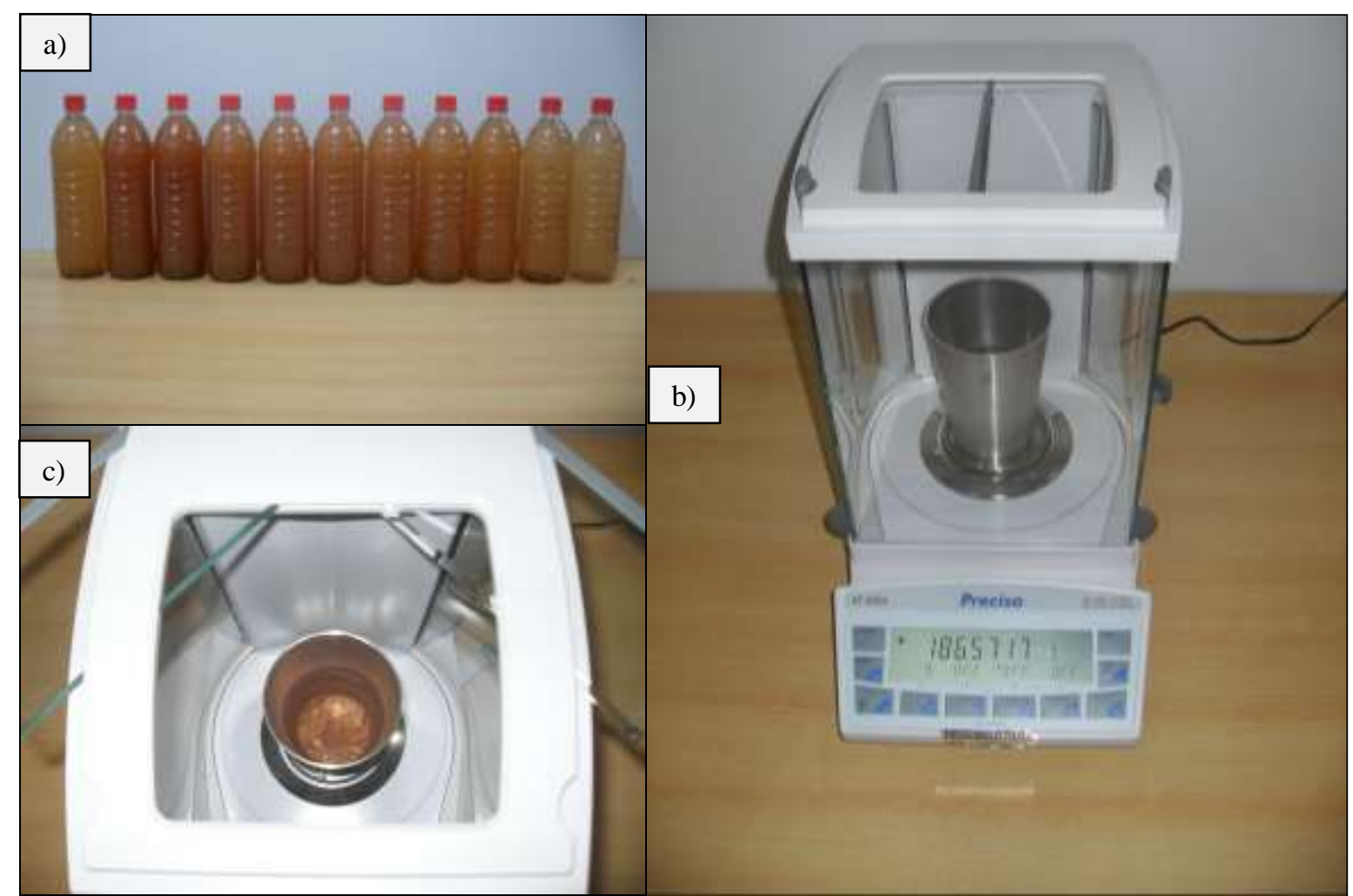

Figura 6.a) Muestras de agua recogidas durante la tormenta del 19-nov-2013; b) Submuestra de agua en balanza de precisión; c) Submuestra ya secada. 
Tabla 2. Ejemplo de cálculo TSD. Muestra $\mathrm{N}^{\circ} 1$ del 19-nov-2013.

\begin{tabular}{|c|c|c|c|c|c|c|c|c|}
\hline \multirow{2}{*}{ Muestra } & \multirow{2}{*}{ Submuestra } & Pr & Ptotal & Ps & Ptotal-Pr & Ps-Pr & \multicolumn{2}{|c|}{ Concentración } \\
\hline & & [gr] & [gr] & [gr] & [gr] & [gr] & \multicolumn{2}{|c|}{ [ppm o mg/l] } \\
\hline & "1-1" & 43.8121 & 184.0741 & 43.8427 & 140.2620 & 0.0306 & 218 & \multirow{3}{*}{185} \\
\hline & "1-2" & 41.0512 & 190.4354 & 41.0772 & 149.3842 & 0.0260 & 174 & \\
\hline & "1-3" & 39.9740 & 185.8181 & 39.9976 & 145.8441 & 0.0236 & 162 & \\
\hline
\end{tabular}

\section{RESULTADOS}

En la campaña de mediciones se alcanzaron los registros de un total de 10 eventos pluviográficos, los cuales se detallan en la Tabla 3 .

Los registros utilizados en esta instancia, poseen las siguientes características:

- Los limnigramas comparados fueron obtenidos con lecturas cada 1 hora.

- Los hietogramas registrados presentan intervalos de tiempo de 5 minutos.

- Todos los eventos poseen registro de las ramas ascendentes y descendentes de los limnigramas.

- Los eventos fueron caracterizados con distintas humedades antecedentes según la metodología propuesta por Chow et al. (1994).

- En todos los eventos se realizaron tomas de muestra de agua para evaluar TSD asociado a cada registro de nivel de agua.

En las Figuras 7 a 16 se exhiben los hietogramas y curvas masa registrados para cada evento, como así también los polutogramas de TSD y los caudales inferidos a través de los limnigramas registrados.

Seguidamente, en la Tabla 4 se exhibe un resumen de los parámetros registrados, como así también los caudales máximos y volúmenes de escurrimiento líquido y sólido inferidos a través de los registros.

Realizando un breve análisis de los resultados, en la Tabla 5 se presentan los valores mínimo, medio y máximo de los parámetros registrados y aquellos inferidos. En la misma puede observarse rangos relativamente amplios de registros, los que cubren un significativo espectro de valores.

Por otro lado, a partir de los valores presentados en la Tabla 4 fue posible construir una relación gráfica entre la producción total de sedimentos por evento observada y la precipitación en exceso. En las Figuras 17 y 18 se exhiben, en el eje "x" la precipitación en exceso o escorrentía en milímetros registrada en los 10 eventos con registros de TSD, mientras que en el eje "y", se presenta el total de sedimentos erogado por la cuenca resultante de la integración de los polutogramas registrados e hidrogramas inferidos a través de los limnigrámas registrados.

La Figura 17 presenta el ploteo y la tendencia de la relación para eventos de 5 a $44 \mathrm{~mm}$ de lluvia neta, la cual exhibe una buena densidad de puntos. Mientras que la Figura 18 incorpora el evento extraordinario del 1-ene-2015 con $126 \mathrm{~mm}$ de precipitación en exceso.

Tabla 3. Características de las tormentas con registros.

\begin{tabular}{|c|c|c|c|c|c|c|}
\hline \multirow[t]{2}{*}{ Evento $\mathbf{N}^{\circ}$} & Fecha & \multirow{2}{*}{$\begin{array}{c}\text { Humedad } \\
\text { antecedente }\end{array}$} & $\begin{array}{c}\text { Precipitación } \\
\text { total }\end{array}$ & Duración & $\begin{array}{c}\text { Intensidad } \\
\text { media }\end{array}$ & $\begin{array}{c}\text { Intensidad máxima en } \\
5 \mathrm{~min}\end{array}$ \\
\hline & [dd-mmm-aa] & & {$[\mathrm{mm}]$} & [h] & {$[\mathbf{m m} / \mathbf{h}]$} & {$[\mathbf{m m} / \mathbf{h}]$} \\
\hline 2 & 11-ene-14 & II (media) & 31.6 & 7 & 4.5 & 24 \\
\hline 3 & $20-\mathrm{feb}-14$ & II (media) & 77.4 & 11 & 7 & 48 \\
\hline 5 & 04-may-14 & II (media) & 38.6 & 10 & 3.9 & 38.4 \\
\hline 6 & 19-jul-14 & II (media) & 58.0 & 12 & 4.8 & 40.8 \\
\hline 7 & 06 -oct-14 & II (media) & 53.8 & 14 & 3.8 & 33.6 \\
\hline 8 & 31 -oct-14 & III (húmedo) & 41.8 & 11 & 3.8 & 33.6 \\
\hline
\end{tabular}



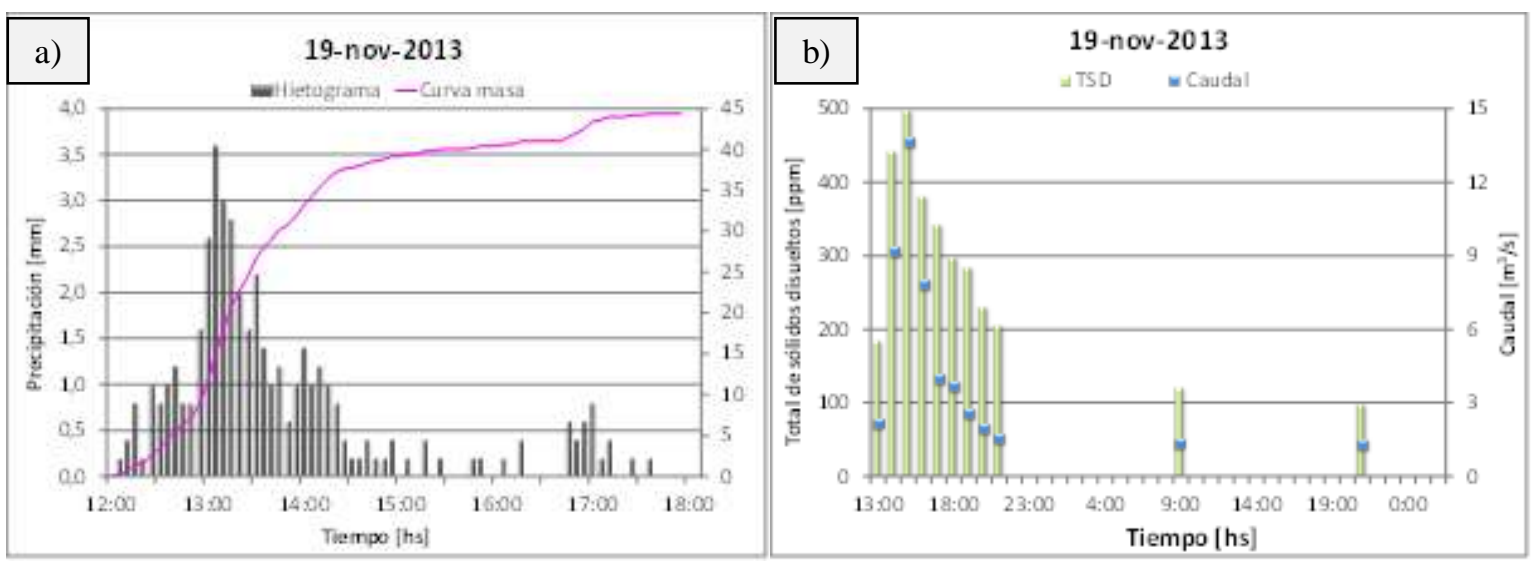

Figura 7. Registros 19-nov-2013. a) Hietograma y curva masa; b) TSD y caudales.
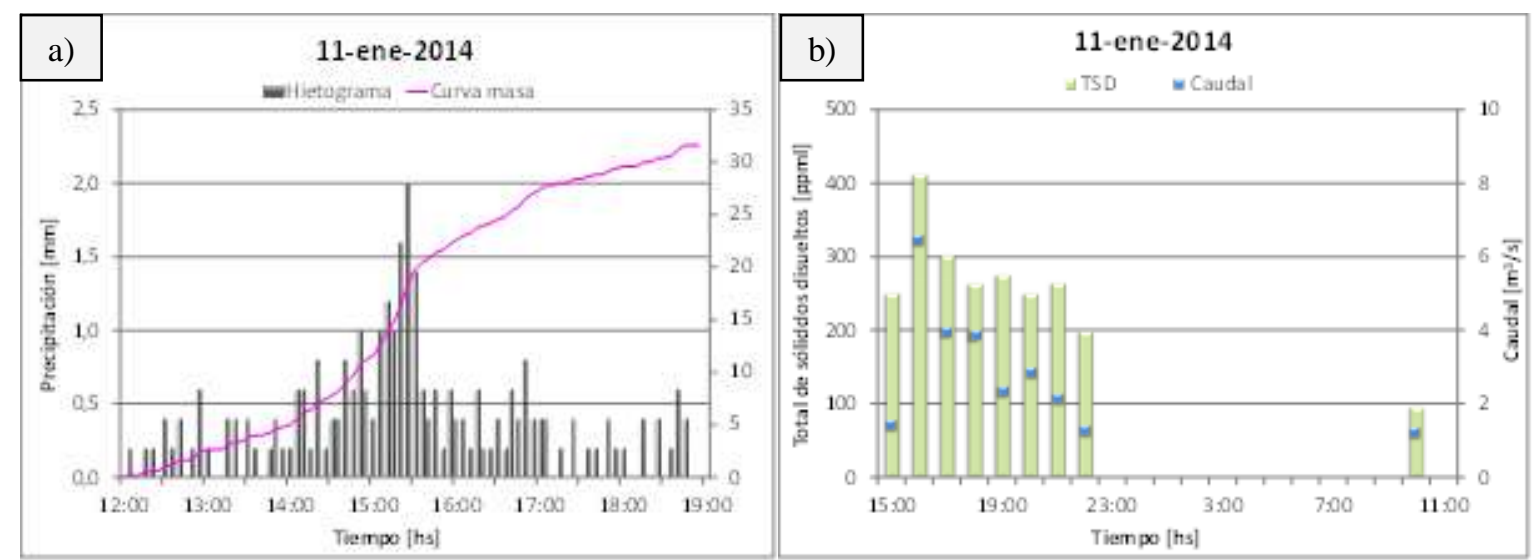

Figura 8. Registros 11-ene-2014. a) Hietograma y curva masa; b) TSD y caudales.
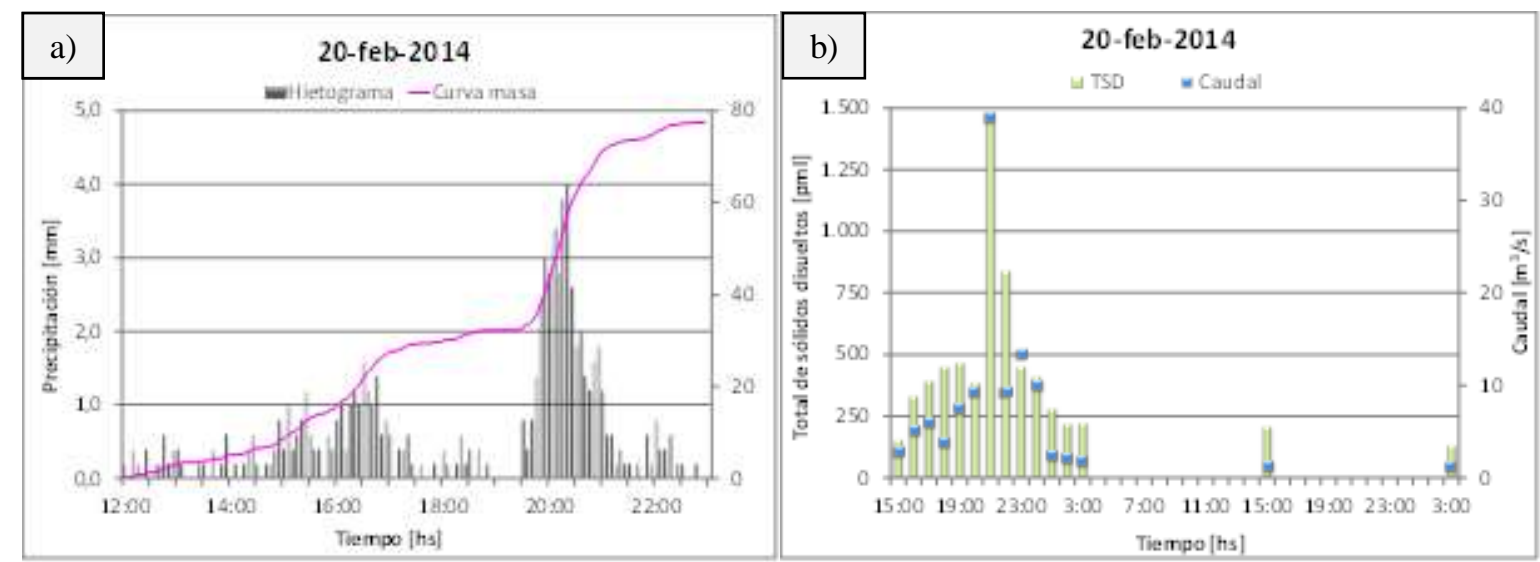

Figura 9. Registros 20-feb-2014. a) Hietograma y curva masa; b) TSD y caudales. 


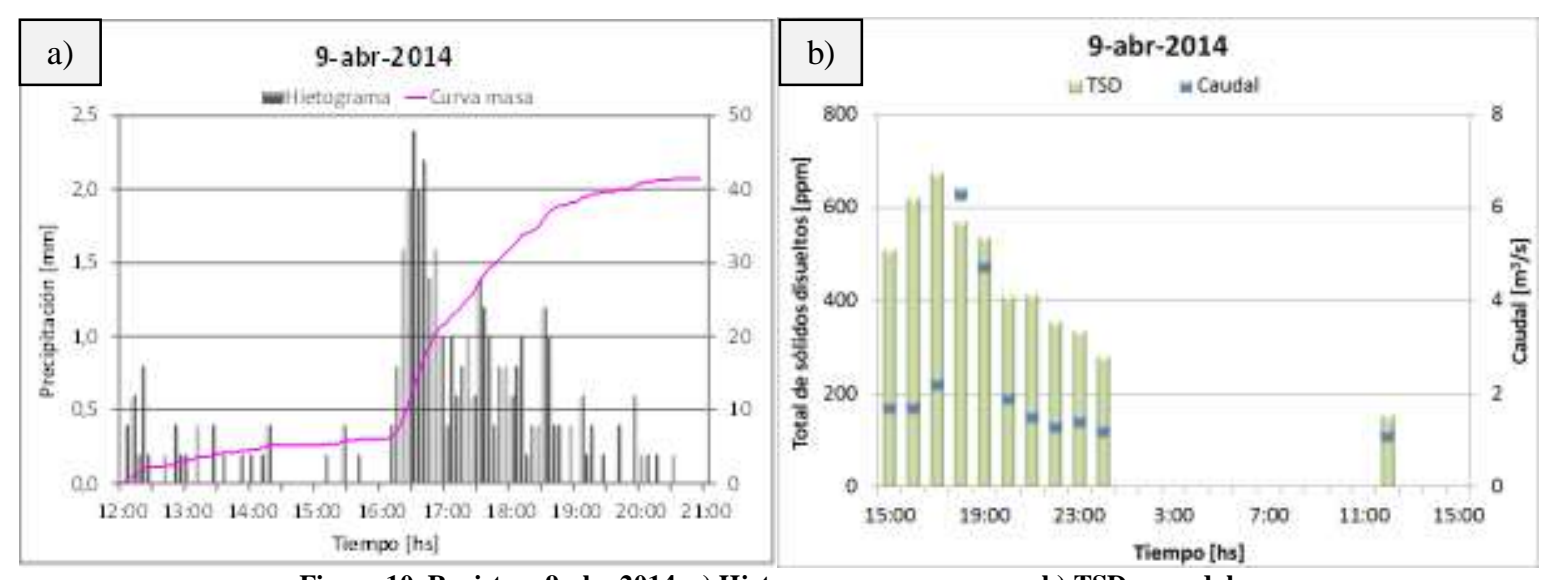

Figura 10. Registros 9-abr-2014. a) Hietograma y curva masa; b) TSD y caudales.
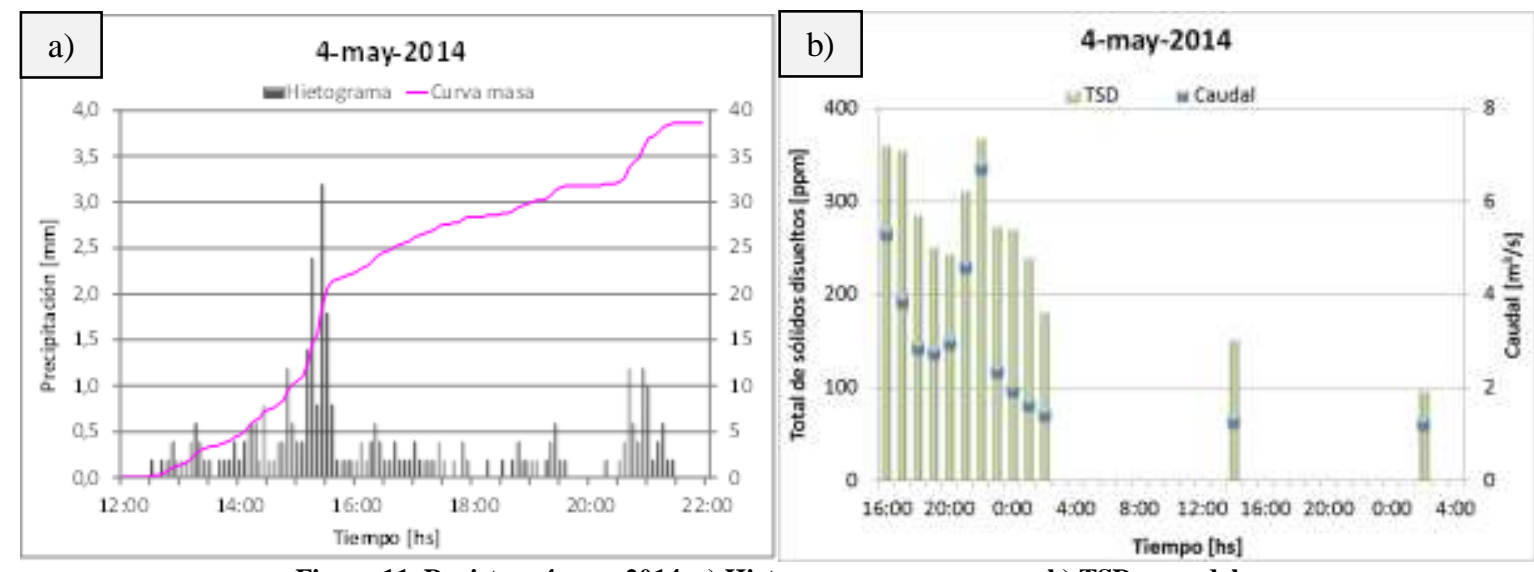

Figura 11. Registros 4-may-2014. a) Hietograma y curva masa; b) TSD y caudales.
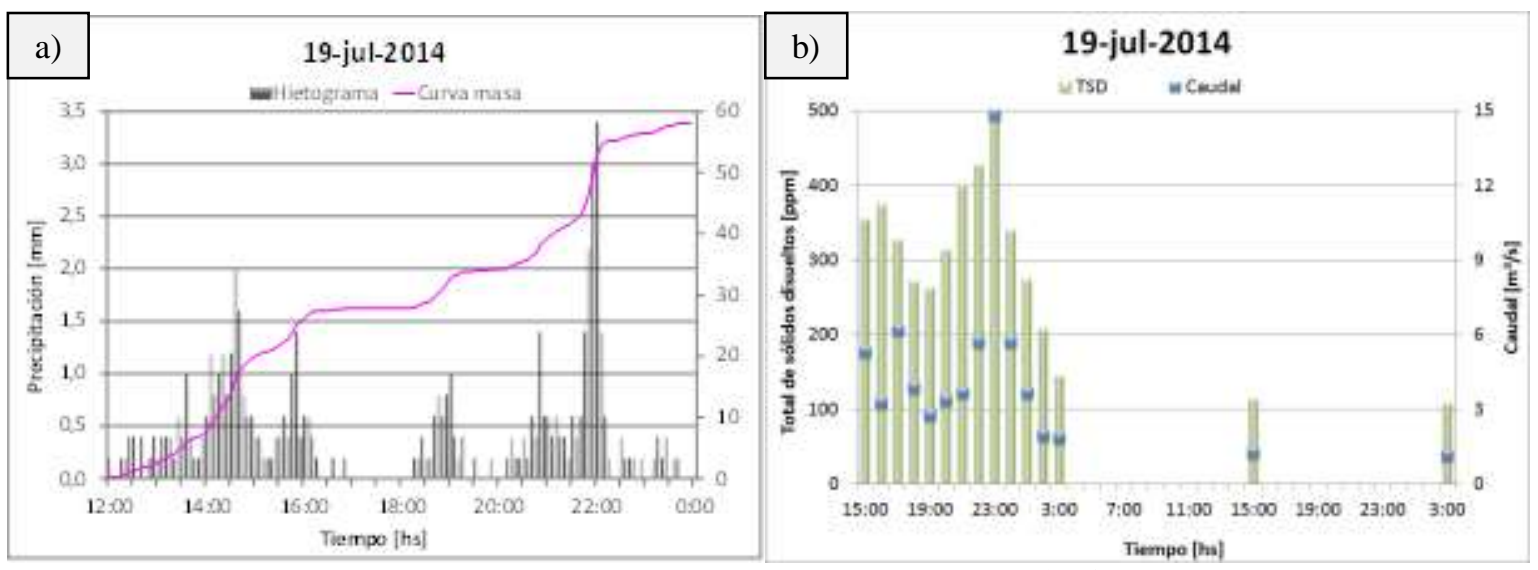

Figura 12. Registros 19-jul-2014. a) Hietograma y curva masa; b) TSD y caudales. 


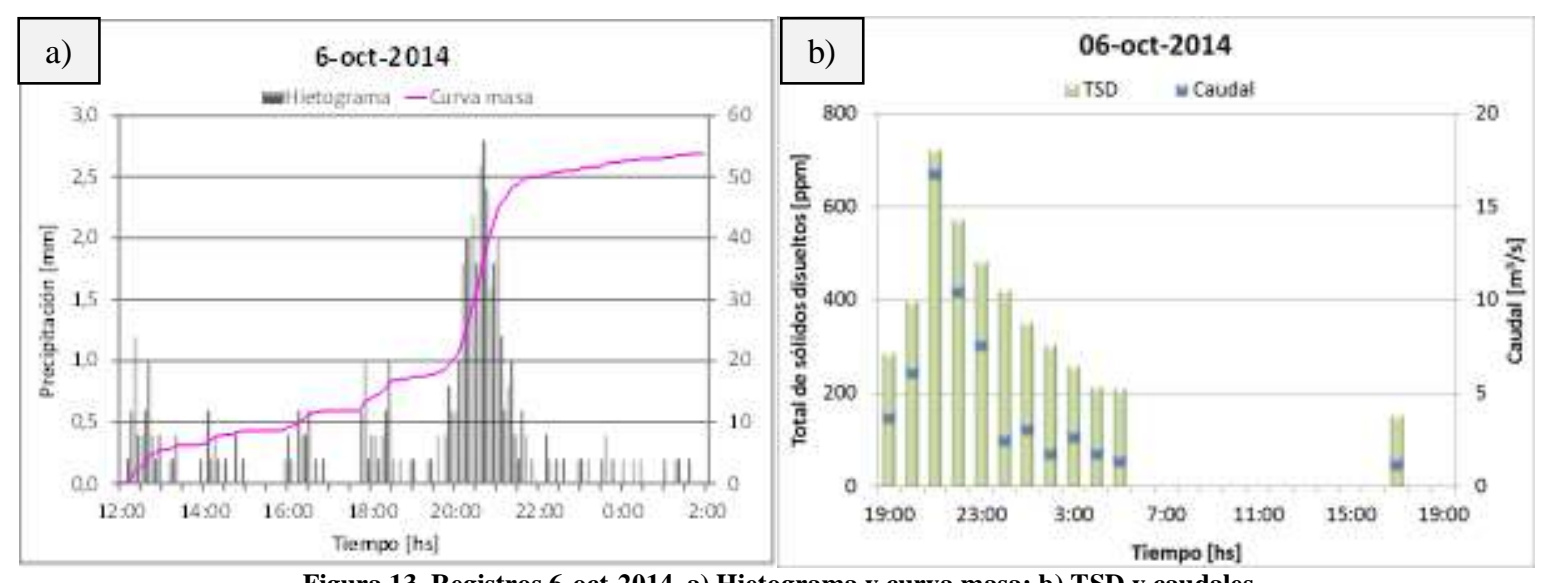

Figura 13. Registros 6-oct-2014. a) Hietograma y curva masa; b) TSD y caudales.
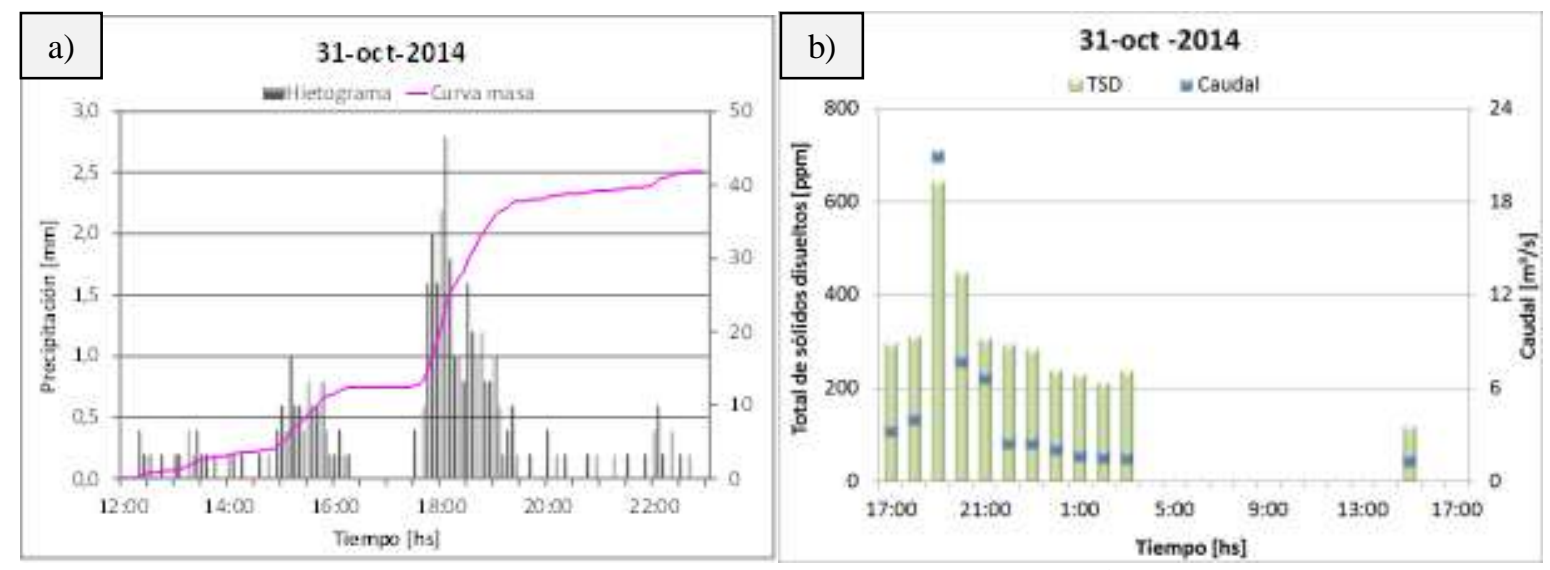

Figura 14. Registros 31-oct-2014. a) Hietograma y curva masa; b) TSD y caudales.
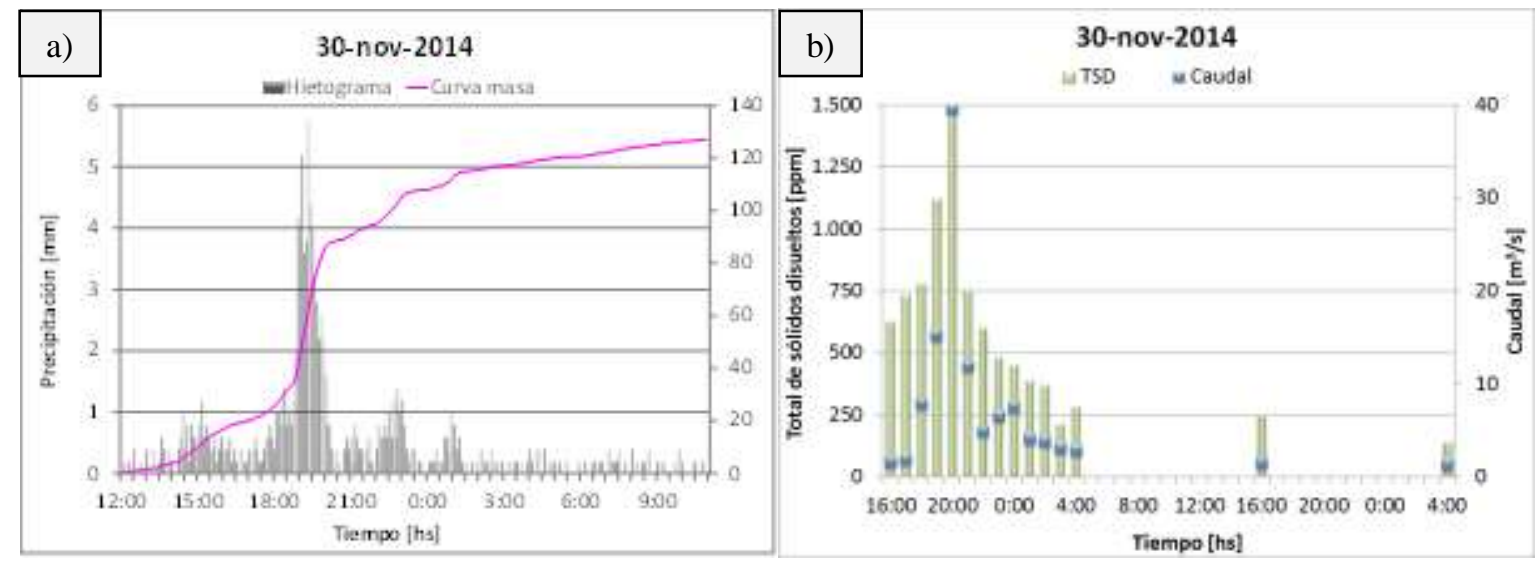

Figura 15. Registros 30-nov-2014. a) Hietograma y curva masa; b) TSD y caudales. 


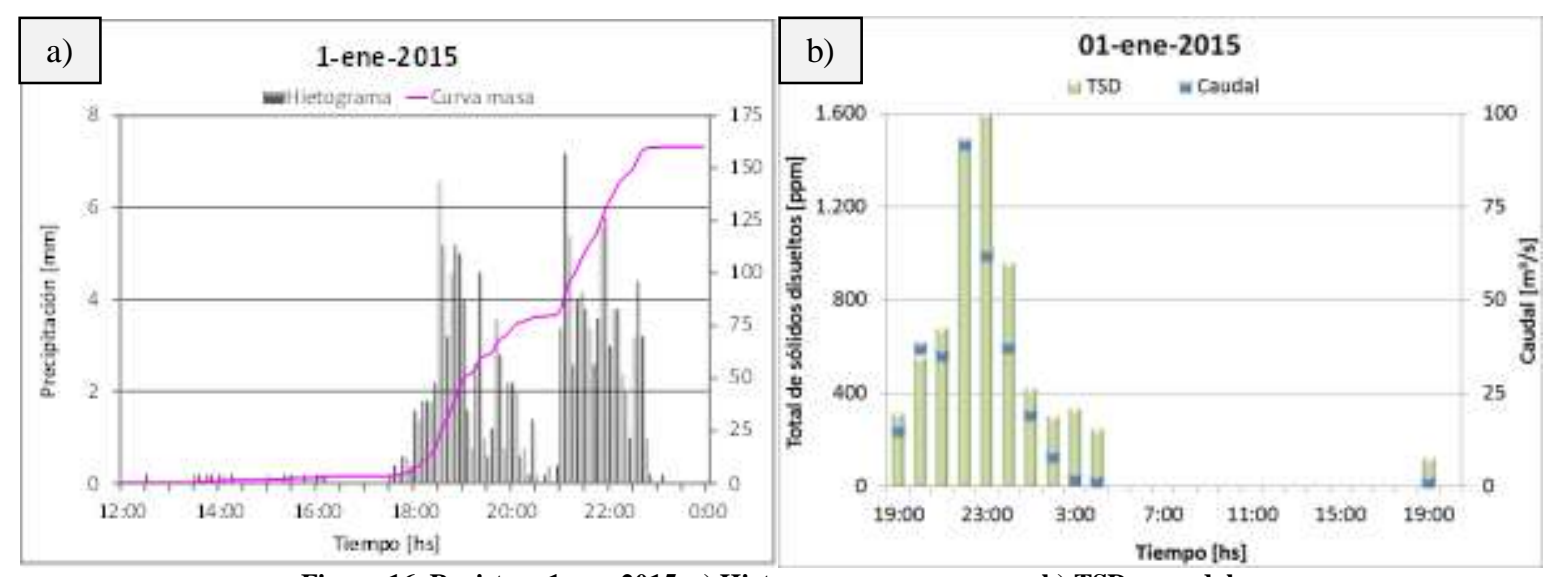

Figura 16. Registros 1-ene-2015. a) Hietograma y curva masa; b) TSD y caudales.

Tabla 4. Resumen de las mediciones realizadas.

\begin{tabular}{|c|c|c|c|c|c|c|c|c|c|c|c|}
\hline & & & & & & & & & & & \\
\hline Evento & {$[-]$} & 1 & 2 & 3 & 4 & 5 & 6 & 7 & 8 & 9 & 10 \\
\hline Lluvia total & {$[\mathrm{mm}]$} & 44.4 & 31.6 & 77.4 & 41.4 & 38.6 & 58 & 53.8 & 41.8 & 127 & 160 \\
\hline Lluvia neta & {$[\mathrm{mm}]$} & 13.6 & 5.2 & 38.0 & 4.6 & 8.5 & 17.5 & 17.6 & 17.2 & 37.2 & 107.5 \\
\hline Duración & [hs] & 6 & 7 & 11 & 9 & 10 & 12 & 14 & 11 & 23 & 12 \\
\hline Humedad previa & {$[-]$} & II & II & II & I & II & II & II & III & I & III \\
\hline Nivel máximo & {$[\mathrm{m}]$} & 1.44 & 1.03 & 2.26 & 1.02 & 1.05 & 1.50 & 1.60 & 1.81 & 2.27 & 2.75 \\
\hline Caudal máximo & {$\left[\mathrm{m}^{3} / \mathrm{s}\right]$} & 15.1 & 6.9 & 43.5 & 6.7 & 7.2 & 16.6 & 19.3 & 25.8 & 43.9 & 69.0 \\
\hline Volumen líquido & {$\left[\mathbf{m}^{3}\right]$} & 132635 & 51031 & 371055 & 45018 & 83165 & 170478 & 172212 & 167656 & 363353 & 1049644 \\
\hline Concentración máxima & [PPM] & 498 & 412 & 1475 & 675 & 369 & 496 & 723 & 645 & 1453 & 1602 \\
\hline Producción total de sedimentos & {$[\mathbf{T}]$} & 89 & 37 & 386 & 52 & 64 & 107 & 132 & 114 & 426 & 1129 \\
\hline
\end{tabular}

Tabla 5. Rango de los parámetros registrados.

\begin{tabular}{|l|c|r|r|r|}
\hline \multicolumn{2}{|c|}{ Parámetro } & Mín. & Medio & \multicolumn{1}{c|}{ Máx. } \\
\hline Lluvia total & {$[\mathrm{mm}]$} & 31.6 & 67.4 & 160 \\
\hline Lluvia neta & {$[\mathrm{mm}]$} & 4.6 & 26.7 & 107.5 \\
\hline Duración & {$[\mathrm{hs}]$} & 6 & 11.5 & 23 \\
\hline Humedad previa & \multicolumn{1}{c|}{$[-]$} & \multicolumn{1}{c|}{ I } & \multicolumn{1}{c|}{ II } & \multicolumn{1}{c|}{ III } \\
\hline Nivel máximo & {$[\mathrm{m}]$} & 1.02 & 1.67 & 2.75 \\
\hline Caudal máximo & {$[\mathrm{m3} / \mathbf{s}]$} & 6.7 & 25.4 & 69.0 \\
\hline Volumen líquido & {$[\mathbf{d m 3}]$} & 45018 & 260625 & 1049644 \\
\hline $\begin{array}{l}\text { Concentración } \\
\text { máxima }\end{array}$ & {$[\mathbf{P P M}]$} & 369 & 835 & 1602 \\
\hline $\begin{array}{l}\text { Producción total de } \\
\text { sedimentos }\end{array}$ & {$[\mathrm{T}]$} & 37 & 254 & 1129 \\
\hline
\end{tabular}

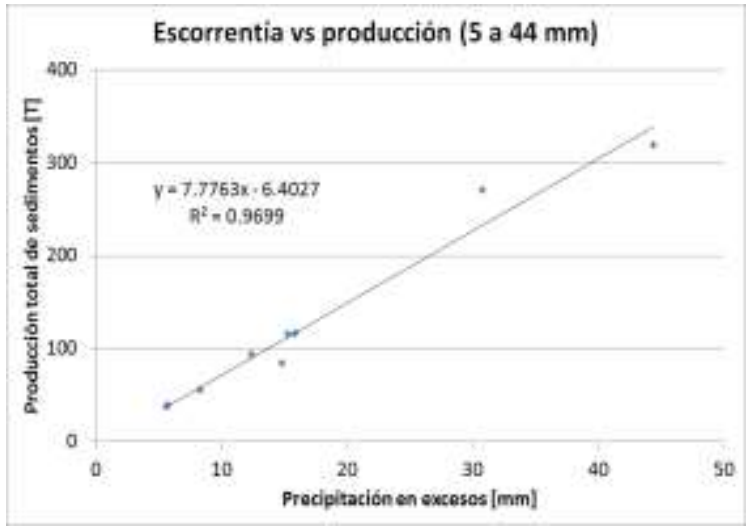

Figura 17. Relación precipitación en exceso vs producción total de sedimentos para eventos de 5 a $44 \mathrm{~mm}$ de lluvia neta. 


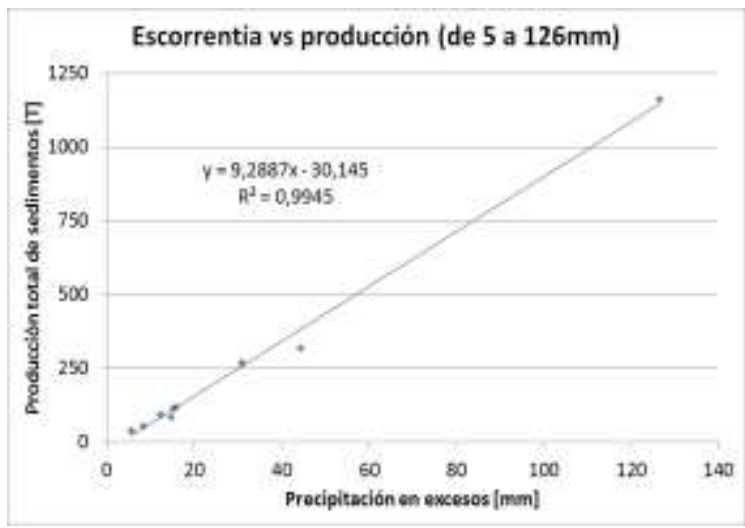

Figura 18. Relación precipitación en exceso vs producción total de sedimentos para eventos de 5 a $126 \mathrm{~mm}$ de lluvia neta.

En la Figura 17 y Figura 18 puede observarse tendencia a relación lineal con $\mathrm{R}^{2}$ cercano a la unidad, representando un buen ajuste.

Finalmente, realizando una comparación entre los caudales máximos y concentraciones de sólidos máximas registrados para cada evento, la misma presenta un mejor ajuste para una ecuación de tipo polinómica, con un R2 de 0.995 (Figura 19).

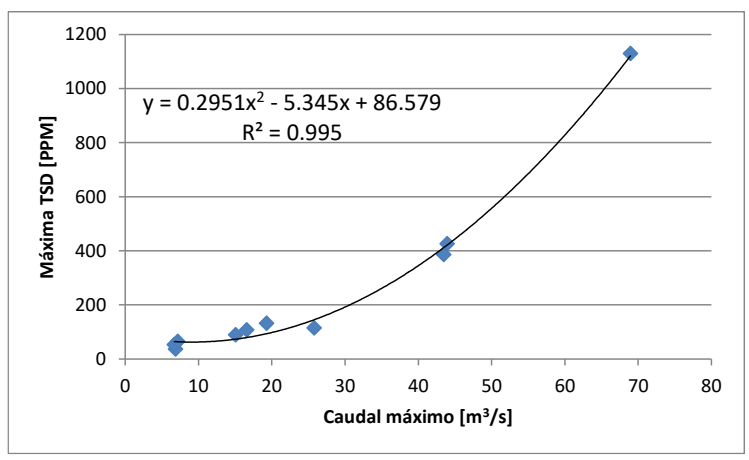

Figura 19. Relación caudales máximos vs concentraciones máximas.

\section{CONCLUSIONES}

La implementación de mediciones de caudal líquido y sólido en la cuenca Mbotaby de la ciudad de Oberá, ha posibilitado avanzar en el estudio de los recursos hídricos superficiales de cuencas urbanas de zona serrana de Misiones.

Si bien 10 tormentas pueden resultar escasas para un proceso de calibración-validación, la medición en campo de cada uno de los eventos implicó un verdadero desafío para el equipo de trabajo del autor, dado que las mismas presentan como una metodología innovadora en la región, desarrolladas generalmente bajo situaciones ambientales adversas.

Las referidas mediciones posibilitarán la calibración y validación de modelos hidrosedimentológicos, permitido así el desarrollo de modernas herramientas de gestión de los desagües pluviales con innumerables aplicaciones futuras.

Como recomendaciones del presente trabajo, surge la necesidad de continuar con los registros durante los eventos pluviográficos a fin de alcanzar la máxima variedad de tormentas posibles de ser evaluadas, considerando la variación de los parámetros de respuesta del sistema en función tanto de la lámina total precipitada como de la distribución temporal de la misma. Un mayor número de tormentas evaluadas permitirá no solo mejorar las técnicas de adquisición y análisis de datos, sino también la calibraciónvalidación de los modelos implementados.

Se considera que las metodologías de medición implementadas en la cuenca Mbotaby, resultan factible de proyectar a otras cuencas urbanas de la ciudad de Oberá y localidades con características fisiográficas y de usos del suelo similares. La medición sistemática de diferentes cuencas piloto en la región, permitiría la concreción de una base de datos disponible para una gestión integral del recurso en la región.

\section{Líneas de trabajo a futuro cercano}

Avanzar en el mejoramiento de la determinación de los campos de velocidades del escurrimiento superficial dentro del dominio espacial de la cuenca, para lo cual se estima la técnica PIV (Particle Image Velocimetry) como pertinente para la medición simultánea dentro del dominio espacial de trabajo y en las condiciones de borde.

Si bien los trabajos de mediciones de producción de sedimentos han sido numerosos e inéditos en la región, se necesita proseguir con tales mediciones dentro del dominio espacial, específicamente en puntos de control internos representativos de los distintos usos y ocupaciones del suelo.

Avanzar hacia una sistematización de las mediciones de escurrimiento líquido y sólido en conjunto con otros organismos universitarios y gubernamentales a fin de aportar más certeza a la descripción de los mecanismos hidrosedimentológicos dominantes. 


\section{REFERENCIAS BIBLIOGRAFICAS}

Compañía Argentina de Relevamiento Topográfico y Aerofotogramétrico (CARTA) (1964). Hoja 2754-25-2D3. Buenos Aires.

Chow, V., Maidment, D. y Mays, L. (1994). Hidrología aplicada. Bogotá, Colombia. Mc Graw-Hill.

Basile, P., Garcia, M. y Riccardi, G. (2011). Calibración de funciones para desagregar en vertical velocidad y concentración de sedimentos en suspensión en el Río Paraná. Memorias del Quinto Simposio Regional sobre Hidráulica de Ríos. Santiago del Estero, Argentina. ISBN 978987-1780-05-1 (Libro + CD-ROM).

Dunne, T. (1986). Urban hydrology in the tropics: problems, solutions, data collection and analysis. en: Urban climatology and its applications with special regard to tropical areas (Proc. WMO technical conference, Mexico DF, November 1984. WMO No 652, WMO, Geneva, Switzerland.

Google Earth @ (2017). Imagen de 11 de diciembre de 2017. www.googleearth.com.

Grupo Multidisciplinar de Modelación de Flujo (GMMF) (2005). Manual del usuario del SWMM 5.0 vE. Universidad Politécnica de Valencia. 244p.

Instituto Nacional de Tecnología Agropecuaria (INTA) Cerro Azul 2013, Comunicación Interna.

Maksimović, Č. (1992). Local floods in urban areas - natural disaster or lack of proper mitigation.
First international conference on disaster prevention in urban areas. Tehran, Iran.

Olinuk, J. (1998). El clima en la localidad de Cerro Azul, período 1967 - 1996. Estación experimental agropecuaria INTA Cerro Azul. Informe técnico $\mathrm{N}^{\circ}$ 68, ISSN 0326-0135. Posadas, Misiones. 73p.

Rodriguez, T. (2018). Estudio de dinámica de los excedentes hídricos superficiales en cuencas urbanas de alta pendiente y sus implicancias en los procesos erosivos. Caso de estudio región urbana de Oberá, Misiones. (Tesis doctoral). Facultad de Ciencia Exactas, Ingeniería y Agrimensura, Universidad Nacional de Rosario. 259p.

Servicio Meteorológico Nacional (SMN) (2018). Boletín climatológico - Anual, Vol. XXIX. ISSN 2314-2332, CABA. 31p.

\section{REGISTRO BIBLIOGRÁFICO:}

Rodriguez, T. y Sanchez, J. (2018). Mediciones de caudal líquido y sólido en cuencas urbanas de zona serrana de Misiones. Cuadernos del CURIHAM. 24, 37-50. DOI: $10.35305 /$ curiham.v24i0.127

\section{Tipo de Publicación: NOTA TÉCNICA.}

Trabajo recibido el 10/07/2018 y aprobado para su publicación el 17/10/2018. 